\title{
LOCAL DERIVATION OF NEST ALGEBRAS
}

\author{
JUN ZHU
}

(Communicated by Palle E. T. Jorgensen)

\begin{abstract}
We show that every strongly continuous local derivation on a nest algebra is a derivation.
\end{abstract}

Let $H$ be a separable and complex Hilbert space, and let $B(H)$ denote the set of all linear bounded operators on $H$. The symbol $x \otimes y$ denotes the rank-1 operator $\langle\cdot, y\rangle x$ for any $x, y \in H$. We identify a closed linear subspace with the corresponding (orthogonal) projection on it. Let $\mathscr{N}$ be a complete nest; then the nest algebra alg $\mathscr{N}$ is the algebra of operators which leave every member of $\mathscr{N}$ invariant. For any $N \in \mathscr{N}$, we define that $N_{-}=\bigvee\{L \in \mathscr{N}: L \subsetneq N\}$ and $N_{+}=\bigwedge\{L \in \mathscr{N}: N \subsetneq L\}$.

If $\mathscr{A}$ is a Banach algebra, we say that a linear transformation $\varphi: \mathscr{A} \rightarrow \mathscr{A}$ is a local derivation if for every $a \in \mathscr{A}$, there is a derivation $\delta_{a}: \mathscr{A} \rightarrow \mathscr{A}$, depending on $a$, such that $\delta_{a}(a)=\varphi(a)$. Larson and Sourour [4] proved that every local derivation on $B(X)$ is a derivation where $X$ is a Banach space. Kadison [3] obtained that every continuous local derivation on a von Neumann algebra is a derivation. We state our main result as follows.

Theorem 1. Let $\varphi$ be a local derivation on a nest algebra which is continuous in the strong operator topology. Then $\varphi$ is a derivation.

First, we prove the following two lemmas.

Lemma 1. Let $\varphi$ be a strong-operator-topology continuous local derivation on a nest algebra $\operatorname{alg} \mathscr{N}$ and let $N$ belong to $\mathscr{N}$ with $N \neq 0$ and $N_{-} \neq H$. Then there are $B_{N}$ and $C_{N}$ in $B(H)$ with $\left.B_{N^{\perp}}\right|_{N^{\perp}}=0$ and $\left.C_{N}\right|_{N_{-}}=0$ such that

(1) $\varphi(x \otimes f)=x \otimes C_{N} f+B_{N} x \otimes f \quad$ for any $x \in N$ and $f \in\left(N_{-}\right)^{\perp}$.

Proof. Note that every derivation on a nest algebra is inner [1]. We may find linear maps $B_{N}$ and $C_{N}$ on $H$ with $\left.B_{N}\right|_{N^{\perp}}=0$ and $\left.C_{N}\right|_{N_{-}}=0$ such that the equality (1) holds, by imitating the proof of Lemmas 1-4 in [4]. So we only need to prove the $B_{N}$ and $C_{N} \in B(H)$. For any $x \in N, f \in\left(N_{-}\right)^{\perp}$, and $u$, $v, w \in H$, we have

$$
\begin{aligned}
\langle\varphi(x \otimes f) u, v\rangle w & =(w \otimes v) \varphi(x \otimes f) u=(w \otimes v)\left(x \otimes C_{N} f+B_{N} x \otimes f\right) u \\
& =\left(\langle x, v\rangle\left\langle u, C_{N} f\right\rangle+\langle u, f\rangle\left\langle B_{N} x, v\right\rangle\right) w .
\end{aligned}
$$

Received by the editors September 15, 1992 and, in revised form, May 24, 1993.

1991 Mathematics Subject Classification. Primary 47D25.

Key words and phrases. Nest algebra, derivation, local derivation. 
So

$$
\langle\varphi(x \otimes f) u, v\rangle=\langle x, v\rangle\left\langle u, C_{N} f\right\rangle+\langle u, f\rangle\left\langle B_{N} x, v\right\rangle .
$$

Fix $u$ and $f$ such that $\langle u, f\rangle \neq 0$. For any $\left\{x_{n}\right\}_{n=1}^{\infty} \subseteq N$ with $x_{n} \stackrel{\|\cdot\|}{\rightarrow} x$ and $B_{N} x_{n} \stackrel{\|\cdot\|}{\rightarrow} y(n \rightarrow \infty)$, we have

$$
\langle\varphi(x \otimes f) u, v\rangle=\langle x, v\rangle\left\langle u, C_{N} f\right\rangle=\langle u, f\rangle\langle y, v\rangle .
$$

By the equalities (2) and (3), we obtain that $\left\langle v, B_{N} x-y\right\rangle=0$ for any $v \in H$, so $B_{N} x=y$, thus $\left.B_{N}\right|_{N}$ is a closed operator. It follows that $B_{N}$ belong to $B(H)$. Similarly, $C_{N} \in B(H)$.

Lemma 2. Let $N_{1}$ and $N_{2} \in \mathscr{N}$ with $N_{1} \subseteq N_{2}, N_{1} \neq 0$, and $\left(N_{2}\right)_{-} \neq H$; and let $\varphi, B_{N_{i}}$, and $C_{N_{i}}(i=1,2)$ be as in Lemma 1. Then there is a $\lambda\left(N_{1}, N_{2}\right) \in \mathbb{C}$ such that

(I) $\varphi(x \otimes f)=x \otimes C_{N_{1}} f+\left[B_{N_{1}}+\lambda\left(N_{1}, N_{2}\right) N_{2}\right] x \otimes f$, and

(II) $\varphi(x \otimes f)=x \otimes\left[C_{N_{1}}+\lambda\left(N_{1}, N_{2}\right)\left(N_{1}\right)_{\perp}^{\perp}\right] f+B_{N_{2}} x \otimes f$

for any $x \in N_{2}, f \in\left(N_{1}\right)_{\perp}^{\perp}$ with $x \otimes f \in \operatorname{alg} \mathscr{N}$.

Proof. Obviously, (I) implies (II), so we only need to prove the equality (I). Let $x \in N_{1}$ and $f \in\left(N_{2}\right)_{-}^{\perp}$. We have $x \otimes f \in \operatorname{alg} \mathscr{N}$ by Lemma 3.3 in [5]. By Lemma 1, we have

$$
x \otimes C_{N_{1}} f+B_{N_{1}} x \otimes f=\varphi(x \otimes f)=x \otimes C_{N_{2}} f+B_{N_{2}} x \otimes f .
$$

So $x \otimes\left(C_{N_{1}}-C_{N_{2}}\right) f=\left(B_{N_{2}}-B_{N_{1}}\right) x \otimes f$; thus there is a $\lambda=\lambda\left(N_{1}, N_{2}\right) \in \mathbb{C}$ such that $\left.\left(C_{N_{1}}-C_{N_{2}}\right)\right|_{\left(N_{2}\right) \perp}=-\lambda\left(N_{2}\right)_{\perp}$ and $\left.\left(B_{N_{1}}-B_{N_{2}}\right)\right|_{N_{1}}=\lambda N_{1}$.

Let $x \otimes f$ be in alg $\mathscr{N}$ where $x \in N_{2}$ and $f \in\left(N_{1}\right)_{-}^{\perp}$. By Lemma 3.3 in [5], there is an $N \in \mathscr{N}$ such that $x \in N$ and $f \in\left(N_{-}\right)^{\perp}$. Without loss of generality, we may assume that $N_{1} \subseteq N \subseteq N_{2}$. Let $B_{N}$ and $C_{N} \in B(H)$ and satisfy the equality (1); as above we may find $\lambda_{i}(i=1,2)$ such that

$$
\begin{array}{ll}
\left.\left(C_{N}-C_{N_{2}}\right)\right|_{\left(N_{2}\right)_{\perp}^{\perp}}=-\lambda_{2}\left(N_{2}\right)_{\perp}^{\perp} ; & \left.\left(B_{N}-B_{N_{2}}\right)\right|_{N}=\lambda_{2} N ; \\
\left.\left(C_{N_{1}}-C_{N}\right)\right|_{\left(N_{-}\right)^{\perp}}=-\lambda_{1}\left(N_{-}\right)^{\perp} ; & \left.\left(B_{N_{1}}-B_{N}\right)\right|_{N_{1}}=\lambda_{1} N_{1} .
\end{array}
$$

Thus

$$
\begin{aligned}
\varphi(x \otimes f) & =x \otimes C_{N} f+B_{N} x \otimes f \\
& =x \otimes\left(C_{N_{1}}+\lambda_{1}\left(N_{-}\right)^{\perp}\right) f+B_{N} x \otimes f \\
& =x \otimes C_{N_{1}} f+\left(B_{N}+\lambda_{1} N\right) x \otimes f \\
& =x \otimes C_{N_{1}} f+\left(B_{N_{2}}+\lambda_{1} N_{2}+\lambda_{2} N_{2}\right) x \otimes f .
\end{aligned}
$$

We only need to prove that $\lambda\left(N_{1}, N_{2}\right)=\lambda_{1}+\lambda_{2}$. For any $x \in N_{1}$ and $f \in N^{\perp}$, using (4) and (5) we see that

$$
\begin{aligned}
& x \otimes C_{N_{1}} f+\left(B_{N_{2}}+\lambda_{1} N_{2}+\lambda_{2} N_{2}\right) x \otimes f \\
& \quad=x \otimes C_{N_{1}} f+B_{N_{1}} x \otimes f=x \otimes C_{N_{1}} f+\left(B_{N_{1}}+\lambda N_{2}\right) x \otimes f .
\end{aligned}
$$

It follows that $\lambda=\lambda\left(N_{1}, N_{2}\right)=\lambda_{1}+\lambda_{2}$ as desired.

Proof of Theorem 1. In the following part, we always assume that $\varphi, C_{N}$, $B_{N}$, and $\lambda\left(N_{1}, N_{2}\right)$ satisfy the equalities (1), (I) and (II) where $\varphi$ is a SOTcontinuous local derivation on $\operatorname{alg} \mathscr{N}$. We claim that there are $B$ and $C$ in 
$B(H)$ such that

$$
\varphi(x \otimes f)=x \otimes C f+B x \otimes f \quad \text { for any } x \otimes f \in \operatorname{alg} \mathscr{N} .
$$

In fact, if $H_{-} \neq H$ and $\{O\}_{+} \neq\{O\}$, we may choose that $C=C_{H}$ and $B=\lambda\left(H,\{O\}_{+}\right)\{O\}_{+}+B_{\{O\}_{+}}$. If $H_{-} \neq H$ and $\{O\}_{+}=\{O\}$, then there is a sequence $\left\{N_{n}\right\}_{n=1}^{\infty} \subseteq \mathcal{N}$ such that $N_{n} \supsetneq N_{n+1}^{*}(n=0,1,2, \ldots), N_{0}=H$, and $\left\{N_{n}\right\}_{n=1}^{\infty}$ converges to $O$ in the strong operator topology. We define that

$$
C_{n}=C_{N_{n}}+\lambda\left(N_{n}, H\right)\left(N_{n}\right)_{-}^{\perp} \quad(n=1,2, \ldots) .
$$

It is clear that $\left.C_{n+1}\right|_{\left(N_{n}\right) \perp}=\left.C_{n}\right|_{\left(N_{n}\right) \perp}$ by Lemma 2 . We claim that $\left\{\left\|C_{n}\right\|: n=\right.$ $1,2, \ldots\}$ be bounded. Suppose that $\sup \left\{\left\|C_{n}\right\|: n=1,2, \ldots\right\}=\infty$; then there are sequences $\left\{n_{k}\right\}_{k=1}^{\infty} \subseteq \mathbb{N}$ and $f_{k} \in\left(N_{n_{k}}\right)_{-}^{\perp}$ with $\left\|f_{k}\right\|=(1 / 2)^{k} \quad(k=$ $1,2, \ldots)$ such that $\left\|C_{n_{k}} f_{k}\right\|>k \quad(k=1,2, \ldots)$. Let $\left\{x_{k}\right\}_{k=1}^{\infty}$ be a unit vector sequence with $x_{k} \in N_{n_{k}}$ and $x_{i} \perp x_{j} \quad(i \neq j)$. We define an operator

$$
T=\lim _{n \rightarrow \infty} \sum_{i=1}^{n} x_{i} \otimes f_{i} \in \operatorname{Alg} \mathscr{N} \text {. }
$$

Thus we have

$$
\begin{aligned}
\left\|\varphi(T) \frac{C_{n_{k}} f_{k}}{\left\|C_{n_{k}} f_{k}\right\|}\right\| & =\left\|\lim _{n \rightarrow \infty} \sum_{i=1}^{n} \varphi\left(x_{i} \otimes f_{i}\right) \frac{C_{n_{k}} f_{k}}{\left\|C_{n_{k}} f_{k}\right\|}\right\| \\
& =\lim _{n \rightarrow \infty}\left\|\sum_{i=1}^{n}\left(x_{i} \otimes C_{n_{i}} f_{i}+B_{H} x_{i} \otimes f_{i}\right) \frac{C_{n_{k}} f_{k}}{\left\|C_{n_{k}} f_{k}\right\|}\right\| \\
& =\lim _{n \rightarrow \infty}\left\|\sum_{i=1}^{n}\left\langle\frac{C_{n_{k}} f_{k}}{\left\|C_{n_{k}} f_{k}\right\|}, C_{n_{i}} f_{i}\right\rangle x_{i}+\left\langle\frac{C_{n_{k}} f_{k}}{\left\|C_{n_{k}} f_{k}\right\|}, f_{i}\right\rangle B_{H} x_{i}\right\| \\
& \geq \lim _{n \rightarrow \infty}\left(\left\|\sum_{i=1}^{n}\left\langle\frac{C_{n_{k}} f_{k}}{\left\|C_{n_{k}} f_{k}\right\|}, C_{n_{i}} f_{i}\right\rangle x_{i}\right\|-\sum_{i=1}^{n} \frac{1}{2^{i}}\left\|B_{H}\right\|\right) \\
& \geq\left\|C_{n_{k}} f_{k}\right\|-\left\|B_{H}\right\|>k-\left\|B_{H}\right\|,
\end{aligned}
$$

contradicting the fact that $\|\varphi(T)\|<\infty$.

It is clear that $\left.C_{n+1}\right|_{\left(N_{n}\right)_{\perp}^{\perp}}=\left.C_{n}\right|_{\left(N_{n}\right)_{\perp}}$ by Lemma 2, so we can define an operator $C$ by $C x=\lim _{n \rightarrow \infty} C_{n} x$ for every $x \in H$ and take $B=B_{H}$. It follows that the operators $B$ and $C$ satisfy the equality (6). The case $H_{-}=H$ can be proved by a similar argument. It follows that the equation (6) holds for any rank-1 operator $x \otimes f \in \operatorname{alg} \mathscr{N}$. By Theorems 1 and 2 of [2], the linear combination of the set $\{x \otimes f: x \otimes f \in$ alg $\mathscr{N}\}$ is dense in the strong operator topology, so we have

$$
\varphi(T)=T C^{*}+B T \quad \text { for any } T \in \operatorname{alg} \mathscr{N} .
$$

In the equality (7), taking $T=I$, we get that $C^{*}+B=0$. Thus $C^{*}=-B$. So $\varphi$ is a derivation.

\section{REFERENCES}

1. E. Christensen, Derivations of nest algebras, Math. Ann. 229 (1977), 155-161.

2. J. A. Erdos, Operators of finite rank in nest algebras, J. London Math. Soc. 43 (1968), 391-397.

3. R. Kadison, Local derivations, preprint. 
4. D. R. Larson and A. R. Sourour, Local derivation and local automorphisms of $B(X)$, Operator Algebras and Applications, Proc. Sympos. Pure Math., vol. 51, Part 2, Amer. Math. Soc., Providence, RI, 1990, pp. 187-194.

5. J. R. Ringrose, On some algebras of operators, Proc. London Math. Soc. (3) 15 (1965), 61-83.

Department of Mathematics, Hubei Institute for Nationalities, Enshi 445000, Peoples REPUBLIC OF CHINA 